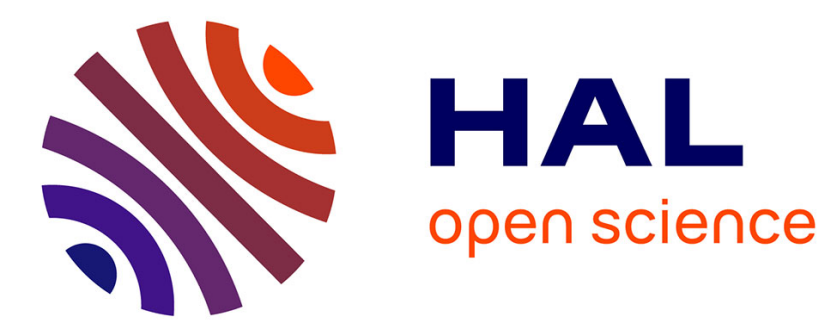

\title{
Optimal control of averaged state of a parabolic equation with missing boundary condition
}

Gisèle Mophou, Romario Gildas Foko Tiomela, Ali Seibou

\section{To cite this version:}

Gisèle Mophou, Romario Gildas Foko Tiomela, Ali Seibou. Optimal control of averaged state of a parabolic equation with missing boundary condition. International Journal of Control, 2018. hal01857372

\section{HAL Id: hal-01857372 \\ https://hal.science/hal-01857372}

Submitted on 15 Aug 2018

HAL is a multi-disciplinary open access archive for the deposit and dissemination of scientific research documents, whether they are published or not. The documents may come from teaching and research institutions in France or abroad, or from public or private research centers.
L'archive ouverte pluridisciplinaire HAL, est destinée au dépôt et à la diffusion de documents scientifiques de niveau recherche, publiés ou non, émanant des établissements d'enseignement et de recherche français ou étrangers, des laboratoires publics ou privés. 


\title{
Optimal control of averaged state of a parabolic equation with missing boundary condition
}

\author{
Gisèle MOPHOU ${ }^{*}$ Romario Gildas FOKO TIOMELA ${ }^{\dagger}$ Ali SEIBOU $\ddagger$
}

August 15, 2018

\begin{abstract}
We consider the optimal control of general heat governed by an operator depend on an unknown parameter and with missing boundary condition. Using the notion of no-regret and low-regret control we prove that we can bring the average of the state of our model to a desired state. Then by means of Euler-Lagrange first order optimality condition, we expressed the optimal control in term of average of an appropriate adjoint state that we characterize by an optimality system. The main tools are the Lebesgue dominated convergence theorem and an appropriate Hilbert space endowed with a norm containing the average of the state.
\end{abstract}

Key-words :No-regret control, Low-regret control, Average control, EulerLagrange first order optimality condition.

AMS Subject Classification 35Q93,49J20, 93C05, 93C41.

\section{Introduction}

Let $N \in \mathbb{N}^{*}$ and $\Omega$ be a bounded open subset of $\mathbb{R}^{N}$ with boundary $\Gamma$ of class $\mathcal{C}^{2}$. Let $\omega$ be an open non-empty subset of $\Omega$. For $T>0$, we set $Q=\Omega \times(0, T)$, $\omega_{T}=\omega \times(0, T)$ and $\Sigma=\Gamma \times(0, T)$. We consider the following controlled parabolic problem:

*African Institute for Mathematical Sciences (AIMS), P.O. Box 608, Limbe Crystal Gardens, South West Region, Cameroon-Laboratoire LAMIA, Université des Antilles, Campus Fouillole, 97159 Pointe-à-Pitre Guadeloupe (FWI)- Laboratoire MAINEGE, Université Ouaga 3S, 06 BP 10347 Ouagadougou 06, Burkina Faso, email : gisele.mophou@univantilles.fr gisele.mophou@aims-cameroon.org

$\dagger$ African Institute for Mathematical Sciences (AIMS), P.O. Box 608, Limbe Crystal Gardens, South West Region, Cameroon, email : romario.foko@aims-cameroon.org

¥Laboratoire MAINEGE, Université Ouaga 3S, 06 BP 10347 Ouagadougou 06, Burkina Faso, email : alichaibou10@gmail.com 


$$
\left\{\begin{aligned}
\frac{\partial y}{\partial t}-\operatorname{div}(\kappa(\alpha, x) \nabla y)+a_{0} y & =f+v \chi_{\omega} & & \text { in } Q \\
y & =g & & \text { on } \Sigma, \\
y(0) & =y^{0} & & \text { in } \Omega,
\end{aligned}\right.
$$

where diffusivity $\kappa \in C([0,1] \times \bar{\Omega})$, the potential $a_{0} \in L^{\infty}(Q)$, the function $f \in L^{2}(Q)$ is given, $v \in L^{2}\left(\omega_{T}\right)$ is the control and $\chi_{\omega}$ is the characteristic function of the control set $\Omega$. The initial condition $y^{0}$ is known and belongs to $L^{2}(\Omega)$. The function $g$ belongs to $L^{2}(\Sigma)$.

Under the assumptions on the data, we know that system (1) has a unique solution $y(\alpha, v, g)=y(\alpha, x, t ; v, g) \in L^{2}(Q)$ such that,

$$
\begin{aligned}
& -\int_{\Omega} y^{0} \varphi(0) d x+\int_{\Sigma} \kappa(\alpha, x) g \frac{\partial \varphi}{\partial \nu} d \sigma d t+\int_{Q} y\left(-\frac{\partial \varphi}{\partial t}-\operatorname{div}(\kappa(\alpha, x) \nabla \varphi)+a_{0} \varphi\right) d x d t \\
& =\int_{Q}\left(f+v \chi_{\omega}\right) \varphi d x d t \\
& \forall \varphi \in \mathcal{C}^{\infty}(\bar{Q}) \text { such that }\left.\varphi\right|_{\Sigma}=0 \text { and } \varphi(T)=0 \text { in } \Omega .
\end{aligned}
$$

System (1) is parameter dependent system with missing boundary condition. We want to act on this system in order to bring the average of the state to a desired state. More precisely, we are interested by the following problem

$$
\inf _{v \in L^{2}\left(\omega_{T}\right)} J(v, g), \quad \forall g \in L^{2}(\Sigma),
$$

where the cost function is given by

$$
J(v, g)=\left\|\int_{0}^{1} y(\alpha, v, g) d \alpha-z_{d}\right\|_{L^{2}(Q)}^{2}+N\|v\|_{L^{2}\left(\omega_{T}\right)}^{2},
$$

with $z_{d} \in L^{2}(Q), N>0$ and $\int_{0}^{1} y(\alpha, v, g) d \alpha \in L^{2}(Q) \forall v \in L^{2}\left(\omega_{T}\right)$ and $\forall g \in$ $L^{2}(\Sigma)$.

As (2) has no sense, we will study this problem combining the notion of noregret and low-regret control introduce by J.L lion [2] to control problem with incomplete data with the notion of averaged control introduce by E. Zuazua [16] to control for parameter dependent system. So we first consider for any $\gamma>0$ the low-regret problem:

$$
\inf _{v \in L^{2}\left(\omega_{T}\right)}\left(\sup _{g \in L^{2}(\Sigma)}\left(J(v, g)-J(0, g)-\gamma\|g\|_{L^{2}(\Sigma)}^{2}\right)\right) .
$$

Then we prove that the low-regret control converge to the no-regret control solution of the problem:

$$
\inf _{v \in L^{2}\left(\omega_{T}\right)}\left(\sup _{g \in L^{2}(\Sigma)}(J(v, g)-J(0, g))\right)
$$


that we characterize by giving the corresponding optimality system.

The notion of no-regret and low regret control was introduced by J. L. Lions [2] to control a parabolic equation governed with an operator free of unknown parameter but with unknown initial condition. According to J.L. Lions, by looking for such a control, one looks for the "best possible control" $v$ which do "at least as well" and "not much worse in the worst situation" than doing nothing. This notion was then applied to control some model with incomplete data, including model involving fractional derivative in time. We refer for instance to $[4,10,11,13,12,14,15]$. The averaged control notion was introduced by $\mathrm{E}$. Zuazua [16] to analyse the problem of controlling parameter dependent systems. In this notion, one is interested by the average of the state with respect to the unknown parameter. for more literature on this topic we refer for instance to $[18,19,17]$ and the reference therein. In this paper we are concerned with the control of a parameter dependent system with missing boundary condition. As far as we know this problem is new.

The rest of this paper is stated as follows. In section 2, we reformulate the low-regret and no-regret control problem (4) and (5). Section 3 is devoted to the proof of the existence of the low-regret control and its characterization. In Section 4, we prove that the low-regret control converges towards the no-regret control that we characterize with an optimality system. Concluding remarks are made in Section 5

\section{Reformulation of the No regret and Low-regret control}

In this section we prove using Legendre-Fenchel transform that the inf-sup problem (4) is equivalent to a classical optimal control problem.

So, let $f \in L^{2}(Q), v \in L^{2}\left(\omega_{T}\right), g \in L^{2}(\Sigma), a_{0} \in L^{\infty}(Q), \kappa \in C([0,1] \times \bar{\Omega})$ and $y^{0} \in L^{2}(\Omega)$. Let also $y(\alpha, v, 0), y(\alpha, 0, g), y(\alpha, 0,0)$ be respectively solutions of:

$$
\begin{gathered}
\left\{\begin{array}{rlll}
\frac{\partial y(\alpha, v, 0)}{\partial t}-\operatorname{div}\left(\kappa(\alpha, x) \nabla y(\alpha, v, 0)+a_{0} y(\alpha, v, 0)\right. & =f+v \chi_{\omega} & \text { in } Q, \\
y(\alpha, v, 0) & =0 & \text { on } \Sigma, \\
y(0 ; \alpha, v, 0) & = & y^{0} & \text { in } \Omega,
\end{array}\right. \\
\left\{\begin{array}{rlll}
\frac{\partial y(\alpha, 0, g)}{\partial t}-\operatorname{div}\left(\kappa(\alpha, x) \nabla y(\alpha, 0, g)+a_{0} y(\alpha, 0, g)\right. & = & f & \text { in } \quad Q, \\
y(\alpha, 0, g) & = & g & \text { on } \Sigma, \\
y(0 ; \alpha, 0, g) & = & y^{0} & \text { in } \Omega
\end{array}\right.
\end{gathered}
$$

and

$$
\left\{\begin{array}{rlll}
\frac{\partial y(\alpha, 0,0)}{\partial t}-\operatorname{div}\left(\kappa(\alpha, x) \nabla y(\alpha, 0,0)+a_{0} y(\alpha, 0,0)\right. & = & f & \text { in } \quad Q \\
y(\alpha, 0,0) & = & 0 & \text { on } \Sigma \\
y(0 ; \alpha, 0,0) & = & y^{0} & \text { in } \Omega .
\end{array}\right.
$$


Then for almost every $\alpha \in[0,1]$, the functions $y(\alpha, v, 0)$ and $y(\alpha, 0,0)$ belong to $L^{2}\left((0, T) ; H_{0}^{1}(\Omega)\right) \cap \mathcal{C}\left([0, T] ; L^{2}(\Omega)\right)$ and we can prove by transposition that $y(\alpha, 0, g)$ belongs to $L^{2}(Q)$ and is such that,

$$
\begin{aligned}
& -\int_{\Omega} y^{0} \varphi(0) d x+\int_{\Sigma} \kappa(\alpha, x) g \frac{\partial \varphi}{\partial \nu} d \sigma d t+\int_{Q} y\left(-\frac{\partial \varphi}{\partial t}-\operatorname{div}(\kappa(\alpha, x) \nabla \varphi)+a_{0} \varphi\right) d x d t \\
& =\int_{Q} f \varphi d x d t, \\
& \forall \varphi \in \mathcal{C}^{\infty}(\bar{Q}) \text { such that }\left.\varphi\right|_{\Sigma}=0 \text { and } \varphi(T)=0 \text { in } \Omega .
\end{aligned}
$$

Remark 1 Note that $\int_{0}^{1} y(\alpha, v, 0) d \alpha, \int_{0}^{1} y(\alpha, 0, g) d \alpha$ and $\int_{0}^{1} y(\alpha, 0,0) d \alpha$ belong to $L^{2}(Q)$ because $\int_{0}^{1} y(\alpha, v, g) d \alpha \in L^{2}(Q)$ for any $v \in L^{2}\left(\omega_{T}\right)$ and $g \in$ $L^{2}(\Sigma)$.

Remark 2 Using Holder inequality, we have that if there exists a constant $C$ independent of $\alpha$ such that $\|y\|_{L^{2}(Q)} \leq C$ then

$$
\left\|\int_{0}^{1} y(\alpha, ., .) d \alpha\right\|_{L^{2}(Q)} \leq C
$$

Lemma 2.1 For any $v \in L^{2}\left(\omega_{T}\right)$ and for any $g \in L^{2}(\Sigma)$, we have:

$$
J(v, g)-J(0, g)=J(v, 0)-J(0,0)+2 \int_{\Sigma}\left(\int_{0}^{1} \kappa(\alpha, x) \frac{\partial \zeta(\alpha, v)}{\partial \nu} d \alpha\right) g d \sigma d t,(9)
$$

where $J$ is the cost function given by $(3)$ and $\zeta(\alpha, v):=\zeta(x, t ; \alpha, v) \in Z^{2,1}(Q)=$ : $L^{2}\left((0, T) ; H^{2}(\Omega) \cap H_{0}^{1}(\Omega)\right) \cap H^{1}\left((0, T) ; L^{2}(\Omega)\right)$ is solution of

$$
\left\{\begin{array}{lll}
-\frac{\partial \zeta(\alpha, v)}{\partial t}-\operatorname{div}\left(\kappa(\alpha, x) \nabla \zeta(\alpha, v)+a_{0} \zeta(\alpha, v)\right. & =-\int_{0}^{1} \eta(\alpha, v) d \alpha & \text { in } \quad Q \\
\zeta(\alpha, v) & =0 & \text { on } \Sigma, \\
\zeta(T ; \alpha, v) & =0 & \text { in } \Omega,
\end{array}\right.
$$

with

$$
\eta(\alpha, v)=y(\alpha, v, 0)-y(\alpha, 0,0)
$$

Remark 3 Note that in view of Remark 2, we have that $\int_{0}^{1} \eta(\alpha, v) d \alpha \in L^{2}(Q)$. 
Proof. Observing that $y(\alpha, v, g)=y(\alpha, v, 0)+y(\alpha, 0, g)-y(\alpha, 0,0)$, we have

$$
\begin{aligned}
J(v, g) & =\left\|\int_{0}^{1}(y(\alpha, v, 0)+y(\alpha, 0, g)-y(\alpha, 0,0)) d \alpha-z_{d}\right\|_{L^{2}(Q)}^{2}+N\|v\|_{L^{2}\left(\omega_{T}\right)}^{2} \\
& =\left\|\int_{0}^{1} y(\alpha, v, 0) d \alpha-z_{d}\right\|_{L^{2}(Q)}^{2}+N\|v\|_{L^{2}\left(\omega_{T}\right)}^{2} \\
& +\left\|\left(\int_{0}^{1} y(\alpha, 0, g) d \alpha-z_{d}\right)-\left(\int_{0}^{1} y(\alpha, 0,0) d \alpha-z_{d}\right)\right\|_{L^{2}(Q)}^{2} \\
& +2 \int_{Q}\left[\int_{0}^{1} y(\alpha, v, 0) d \alpha-z_{d}\right]\left[\int_{0}^{1}(y(\alpha, 0, g)-y(\alpha, 0,0)) d \alpha\right] d x d t \\
& =J(v, 0)+\left\|\int_{0}^{1} y(\alpha, 0, g) d \alpha-z_{d}\right\|_{L^{2}(Q)}^{2}+\left\|\int_{0}^{1} y(\alpha, 0,0) d \alpha-z_{d}\right\|_{L^{2}(Q)}^{2} \\
& -2 \int_{Q}\left[\int_{0}^{1} y(\alpha, 0, g) d \alpha-z_{d}\right]\left[\int_{0}^{1} y(\alpha, 0,0) d \alpha-z_{d}\right] d x d t \\
& +2 \int_{Q}\left[\int_{0}^{1}(y(\alpha, v, 0)-y(\alpha, 0,0)) d \alpha\right]\left[\int_{0}^{1}(y(\alpha, 0, g)-y(\alpha, 0,0)) d \alpha\right] d x d t \\
& +2 \int_{Q}\left[\int_{0}^{1}\left(y(\alpha, 0,0) d \alpha-z_{d}\right)\right]\left[\int_{0}^{1}(y(\alpha, 0, g)-y(\alpha, 0,0)) d \alpha\right] d x d t \\
& =J(v, 0)+J(0, g)-J(0,0) \\
& +2 \int_{Q}\left[\int_{0}^{1}(y(\alpha, v, 0)-y(\alpha, 0,0)) d \alpha\right]\left[\int_{0}^{1}(y(\alpha, 0, g)-y(\alpha, 0,0)) d \alpha\right] d x d t
\end{aligned}
$$

Consequently,

$$
\begin{aligned}
& J(v, g)-J(0, g)=J(v, 0)-J(0,0)+ \\
& 2 \int_{Q}\left[\int_{0}^{1}(y(\alpha, v, 0)-y(\alpha, 0,0)) d \alpha\right]\left[\int_{0}^{1}(y(\alpha, 0, g)-y(\alpha, 0,0)) d \alpha\right] d x d t .
\end{aligned}
$$

In view of Remark 3, system (10) has a unique solution $\zeta(\alpha, v) \in Z^{2,1}(Q)$. Moreover, there exists a constant $C>0$ independent of $\alpha$ such that

$$
\|\zeta(\alpha, v)\|_{L^{2}\left((0, T) ; H^{2}(\Omega)\right)} \leq C\|v\|_{L^{2}\left(\omega_{T}\right)} .
$$

Therefore, using the continuity of the trace operator, we have that

$$
\left\|\frac{\partial \zeta(\alpha, v)}{\partial \nu}\right\|_{L^{2}(\Sigma)} \leq C\|v\|_{L^{2}\left(\omega_{T}\right)}
$$

We set

$$
\pi(\alpha, g)=y(\alpha, 0, g)-y(\alpha, 0,0)
$$


In view of Remark 2, we have that $\int_{0}^{1} \pi(\alpha, g) d \alpha \in L^{2}(Q)$. Observing that $\pi(\alpha, g)$ is solution of

$$
\left\{\begin{array}{rlll}
\frac{\partial \pi}{\partial t}-\operatorname{div}(\kappa(\alpha, x) \nabla \pi)+a_{0} \pi & = & \text { in } \quad Q \\
\pi & =g & \text { on } \Sigma \\
\pi(0) & = & \text { in } \Omega
\end{array}\right.
$$

if we multiply the first equation of (10) by $\int_{0}^{1} \pi(\alpha, g) d \alpha$ and we integrate over $Q$, we have:

$$
\begin{aligned}
& -\int_{Q}\left[\int_{0}^{1}(y(\alpha, v, 0)-y(\alpha, 0,0)) d \alpha\right]\left[\int_{0}^{1}(y(\alpha, 0, g)-y(\alpha, 0,0)) d \alpha\right] d x d t \\
& =\int_{0}^{1}\left[\int_{Q}\left(-\frac{\partial \zeta(\alpha, v)}{\partial t}-\operatorname{div}\left(\kappa(\alpha, x) \nabla \zeta(\alpha, v)+a_{0} \zeta(\alpha, v)\right) \pi(\alpha, g) d x d t\right] d \alpha\right. \\
& =-\int_{\Sigma}\left(\int_{0}^{1} \kappa(\alpha, x) \frac{\partial \zeta(\alpha, v)}{\partial \nu} d \alpha\right) g d \sigma d t
\end{aligned}
$$

Hence,

$$
\begin{aligned}
& \int_{Q}\left[\int_{0}^{1}(y(\alpha, v, 0)-y(\alpha, 0,0)) d \alpha\right]\left[\int_{0}^{1}(y(\alpha, 0, g)-y(\alpha, 0,0)) d \alpha\right] d x d t \\
= & \int_{\Sigma}\left(\int_{0}^{1} \kappa(\alpha, x) \frac{\partial \zeta(\alpha, v)}{\partial \nu} d \alpha\right) g d \sigma d t .
\end{aligned}
$$

If we combine (16) and (12), we obtain that

$$
J(v, g)-J(0, g)=J(v, 0)-J(0,0)+2 \int_{\Sigma}\left(\int_{0}^{1} \kappa(\alpha, x) \frac{\partial \zeta(\alpha, v)}{\partial \nu} d \alpha\right) g d \sigma d t .
$$

Remark 4 As $\kappa \in C([0,1] \times \bar{\Omega})$, using (14), we have that $\kappa \frac{\partial \zeta(\alpha, v)}{\partial \nu}$ is bounded in $L^{2}(\Sigma)$ independently of $\alpha$. It then follows from Remark 1 that $\int_{0}^{1} \kappa(\alpha, x) \frac{\partial \zeta(\alpha, v)}{\partial \nu} d \alpha \in$ $L^{2}(\Sigma)$. This means that (9) has a sense.

From now on, we set

$$
\frac{\partial \tilde{\zeta}(\alpha, v)}{\partial \nu}=\int_{0}^{1} \kappa(\alpha, x) \frac{\partial \zeta(\alpha, v)}{\partial \nu} d \alpha .
$$

Then according to (9), problem (5) is equivalent to:

$$
\inf _{v \in L^{2}\left(\omega_{T}\right)}\left(\sup _{g \in L^{2}(\Sigma)}\left(J(v, 0)-J(0,0)+2 \int_{\Sigma} \frac{\partial \tilde{\zeta}(\alpha, v)}{\partial \nu} g d \sigma d t\right)\right) .
$$


Consequently, the no-regret control belongs to the set

$$
\mathcal{O}=\left\{v \in L^{2}\left(\omega_{T}\right) \quad \text { such that } \frac{\partial \tilde{\zeta}(\alpha, v)}{\partial \nu} \text { is orthogonal to } L^{2}(\Sigma)\right\} .
$$

As such a control is not easy to characterize, we consider the low-regret control problem. Using (9), problem (4) could be rewritten as

$$
\begin{aligned}
& \inf _{v \in L^{2}\left(\omega_{T}\right)}(J(v, 0)-J(0,0) \\
& \left.+2 \sup _{g \in L^{2}(\Sigma)}\left(\int_{\Sigma} \frac{\partial \tilde{\zeta}(\alpha, v)}{\partial \nu} g d \sigma d t-\frac{\gamma}{2}\|g\|_{L^{2}(\Sigma)}^{2}\right)\right),
\end{aligned}
$$

which by means of Legendre-Fenchel transform is equivalent to the following problem: For any $\gamma>0$, find $u^{\gamma} \in L^{2}(Q)$ such that

$$
J_{\gamma}\left(u^{\gamma}\right)=\inf _{v \in L^{2}\left(\omega_{T}\right)} J_{\gamma}(v)
$$

where

$$
J_{\gamma}(v)=J(v, 0)-J(0,0)+\frac{1}{\gamma}\left\|\frac{\partial \tilde{\zeta}(\alpha, v)}{\partial \nu}\right\|_{L^{2}(\Sigma)}^{2}
$$

\section{Existence and characterization of the low-regret control}

Our main objective in this section will be to show that the low-regret problem (20) has a unique solution which converges to the no-regret control unique solution of (18). Furthermore, we will give the equations that characterize the low-regret control.

Remark 5 From now on, we use $C(X)$ to denote a positive constant whose value varies from a line to another but depends on $X$.

Proposition 3.1 There exists a unique low-regret control $u^{\gamma} \in L^{2}\left(\omega_{T}\right)$ solution of (20).

Proof. Observing that We have:

$$
-J(0,0) \leq J(v, 0)-J(0,0)+\frac{1}{\gamma}\left\|\int_{0}^{1} \kappa(\alpha, x) \frac{\partial \zeta(\alpha, v)}{\partial \nu} d \alpha\right\|_{L^{2}(\Sigma)}^{2} \quad \forall v \in L^{2}\left(\omega_{T}\right),
$$

we have that the set $\left\{J_{\gamma}(v), J_{\gamma}(v) \geq-J(0,0), v \in L^{2}\left(\omega_{T}\right)\right\}$ is not empty. Consequently, there exists $m_{\gamma}$ such that

$$
m_{\gamma}=\inf _{v \in L^{2}\left(\omega_{T}\right)} J_{\gamma}(v)
$$


Let $v_{n} \in L^{2}\left(\omega_{T}\right)$ be a minimizing sequence such that

$$
J_{\gamma}\left(v_{n}\right) \longrightarrow m_{\gamma} .
$$

Then, $y_{n}:=y\left(x, t ; \alpha, v_{n}, 0\right)$ satisfies:

$$
\left\{\begin{aligned}
\frac{\partial y_{n}}{\partial t}-\operatorname{div}\left(\kappa(\alpha, x) \nabla y_{n}\right)+a_{0} y_{n} & =f+v_{n} \chi_{\omega} & & \text { in } Q \\
y_{n} & =0 & & \text { on } \Sigma \\
y_{n}(0) & =y^{0} & & \text { in } \Omega .
\end{aligned}\right.
$$

Let us set $\tilde{\zeta}_{n}=\tilde{\zeta}\left(v_{n}\right)=\int_{0}^{1} \kappa(\alpha, x) \frac{\partial \zeta\left(\alpha, v_{n}\right)}{\partial \nu} d \alpha$ where $\zeta_{n}=\zeta\left(\alpha, v_{n}\right)$ is solution of (10).

In view of (22), there exists a constant $C>0$ independent of $n$ and $\gamma$ such that $-J(0,0) \leq J_{\gamma}\left(v_{n}\right) \leq C$ which implies:

$$
0 \leq J\left(v_{n}, 0\right)+\frac{1}{\gamma}\left\|\frac{\partial \tilde{\zeta}_{n}}{\partial \nu}\right\|_{L^{2}(\Sigma)}^{2} \leq C+J(0,0)
$$

Hence there exists a non negative constant $C$ independent of $n$ and $\gamma$, such that:

$$
0 \leq J\left(v_{n}, 0\right)+\frac{1}{\gamma}\left\|\frac{\partial \tilde{\zeta}_{n}}{\partial \nu}\right\|_{L^{2}(\Sigma)}^{2} \leq C
$$

which implies that

$$
\begin{aligned}
J\left(v_{n}, 0\right) & \leq C \\
\frac{1}{\gamma}\left\|\frac{\partial \tilde{\zeta}_{n}}{\partial \nu}\right\|_{L^{2}(\Sigma)}^{2} & \leq C .
\end{aligned}
$$

Hence, using the fact that

$$
J\left(v_{n}, 0\right)=\left\|\int_{0}^{1} y\left(\alpha, v_{n}, 0\right) d \alpha-z_{d}\right\|_{L^{2}(Q)}^{2}+N\left\|v_{n}\right\|_{L^{2}\left(\omega_{T}\right)}^{2},
$$

we deduce that

$$
\begin{aligned}
\left\|v_{n}\right\|_{L^{2}\left(\omega_{T}\right)} & \leq C \\
\left\|\frac{\partial \tilde{\zeta}_{n}}{\partial \nu}\right\|_{L^{2}(\Sigma)} & \leq C \sqrt{\gamma} \\
\left\|\int_{0}^{1} y_{n}(\alpha, . .,) d \alpha\right\|_{L^{2}(Q)} & \leq C .
\end{aligned}
$$

In view of (23) and (24), there exists $C>0$ independent of $n$ and $\gamma$ such that

$$
\left\|y_{n}\right\|_{L^{2}\left((0, T) ; H_{0}^{1}(\Omega)\right)} \leq C
$$


Hence, there exist $u^{\gamma} \in L^{2}\left(\omega_{T}\right), y^{\gamma} \in L^{2}\left((0, T) ; H_{0}^{1}(\Omega)\right)$ and subsequences extracted from $\left(v_{n}\right)$ and $\left(y_{n}\right)$ (still denoted $\left(v_{n}\right)$ and $\left.\left(y_{n}\right)\right)$ such that

$$
\begin{array}{lll}
v_{n} \rightarrow u^{\gamma} & \text { weakly in } L^{2}\left(\omega_{T}\right), \\
y_{n} \rightarrow y^{\gamma} & \text { weakly in } L^{2}\left((0, T) ; H_{0}^{1}(\Omega)\right) .
\end{array}
$$

We set $z_{n}(x, t)=\int_{0}^{1} y_{n}(\alpha, x, t) d \alpha$. Then in view of $(26)$ there exist $z^{\gamma} \in L^{2}(Q)$ such that

$$
\begin{aligned}
& \int_{Q} z_{n}(x, t) \varphi(x, t) d x d t=\int_{0}^{1}\left(\int_{Q} y_{n}(x, t) \varphi(x, t) d x d t\right) \rightarrow \int_{Q} z^{\gamma}(x, t) \varphi(x, t) d x d t \\
& \forall \varphi \in L^{2}(Q) .
\end{aligned}
$$

On the other hand, using the fact that $y_{n}$ is bounded independently of $\alpha$ and the fact that

$$
\int_{Q} y_{n}(x, t) \varphi(x, t) d x d t \rightarrow \int_{Q} y^{\gamma}(x, t) \varphi(x, t) d x d t \quad \forall \varphi \in L^{2}(Q),
$$

it follows from the Lebesgue dominated convergence theorem that

$$
\int_{0}^{1}\left[\int_{Q} y_{n}(\alpha, x, t) \varphi(x, t) d x d t\right] d \alpha=\int_{Q}\left[\int_{0}^{1} y_{n}(\alpha, x, t) d \alpha\right] \varphi(x, t) d x d t
$$

converges towards

$$
\int_{0}^{1}\left[\int_{Q} y^{\gamma}(\alpha, x, t) \varphi(x, t) d x d t\right] d \alpha=\int_{Q}\left[\int_{0}^{1} y^{\gamma}(\alpha, x, t) d \alpha\right] \varphi(x, t) d x d t .
$$

Therefore, from (30) and the uniqueness of the limit, we have that

$$
\int_{0}^{1} y^{\gamma}(\alpha, x, t) d \alpha=z^{\gamma}(x, t)
$$

We thus can write

$$
\int_{Q} \int_{0}^{1} y\left(\alpha, v_{n}, 0\right) \varphi d \alpha d x d t \rightarrow \int_{Q} \int_{0}^{1} y\left(\alpha, u^{\gamma}, 0\right) \varphi(x, t) d \alpha d x d t .
$$

The rest of the proof will be divided into steps.

Step 1. We prove that $\left(u^{\gamma}, y^{\gamma}\right)$ satisfies (6).

Let $\mathcal{D}(Q)$ being the set of functions of class $C^{\infty}$ on $Q$ with compact support. Multiplying the first equation in $(23)$ by $\varphi \in \mathcal{D}(Q)$ and integrating by parts over Q,

$$
\int_{Q} y_{n}\left(-\frac{\partial \varphi}{\partial t}-\operatorname{div}(\kappa(\alpha, x) \nabla \varphi)+a_{0} \varphi\right) d x d t=\int_{Q}\left(f+v_{n} \chi_{\omega}\right) \varphi d x d t
$$


Passing this latter identity to the limit when $n \rightarrow \infty$ while using (28) and (29), we obtain that

$$
\int_{Q} y^{\gamma}\left(-\frac{\partial \varphi}{\partial t}-\operatorname{div}(\kappa(\alpha, x) \nabla \varphi)+a_{0} \varphi\right) d x d t=\int_{Q}\left(f+u^{\gamma} \chi_{\omega}\right) \varphi d x d t
$$

which after integration by parts gives over $Q$,

$$
\int_{Q}\left(\frac{\partial y^{\gamma}}{\partial t}-\operatorname{div}\left(\kappa(\alpha, x) \nabla y^{\gamma}\right)+a_{0} y^{\gamma}\right) \varphi d x d t=\int_{Q}\left(f+u^{\gamma} \chi_{\omega}\right) \varphi d x d t .
$$

Hence, we deduce that

$$
\frac{\partial y^{\gamma}}{\partial t}-\operatorname{div}\left(\kappa(\alpha, x) \nabla y^{\gamma}\right)+a_{0} y^{\gamma}=f+u^{\gamma} \chi_{\omega} \text { in } Q .
$$

Now, as, $y^{\gamma} \in L^{2}\left((0, T), H_{0}^{1}(\Omega)\right)$, using (34), we have that $\frac{\partial y^{\gamma}}{\partial t} \in L^{2}\left((0, T), H^{-1}(\Omega)\right)$. This implies that $y^{\gamma} \in \mathcal{C}\left([0, T], L^{2}(\Omega)\right)$. On the other hand, $y^{\gamma} \in L^{2}(Q)$ and $\operatorname{div}\left(\kappa(\alpha, x) \nabla y^{\gamma}\right) \in H^{-1}\left((0, T), L^{2}(\Omega)\right)$. This implies that $\left.y^{\gamma}\right|_{\Sigma}$ and $\left.\frac{\partial y^{\gamma}}{\partial \nu}\right|_{\Sigma}$ exist and belong respectively to $H^{-1}\left((0, T) ; H^{-\frac{1}{2}}(\Gamma)\right)$ and $H^{-1}\left((0, T) ; H^{-\frac{3}{2}}(\Gamma)\right)$.

Now, let $\varphi \in \mathcal{C}^{\infty}(\bar{Q})$ with $\left.\varphi\right|_{\Sigma}=0$ and $\varphi(T)=0$ in $\Omega$. If we multiply the first equation in (23) by $\varphi$ and we integrate over $Q$, we obtain:

$$
\begin{aligned}
\int_{0}^{T} \int_{\Omega}\left(f+v_{n} \chi_{\omega}\right) \varphi d x d t & =-\int_{\Omega} y^{0} \varphi(x, 0) d x \\
& +\int_{0}^{T} \int_{\Omega} y_{n}\left(-\frac{\partial \varphi}{\partial t}-\operatorname{div}(\kappa(\alpha, x) \nabla \varphi)+a_{0} \varphi\right) d x d t
\end{aligned}
$$

which by passing to the limit when $n \longrightarrow \infty$ while using (28) and (29) gives

$$
\begin{aligned}
\int_{0}^{T} \int_{\Omega}\left(f+u^{\gamma} \chi_{\omega}\right) \varphi d x d t & =-\int_{\Omega} y^{0} \varphi(x, 0) d x \\
& +\int_{0}^{T} \int_{\Omega} y^{\gamma}\left(-\frac{\partial \varphi}{\partial t}-\operatorname{div}(\kappa(\alpha, x) \nabla \varphi)+a_{0} \varphi\right) d x d t \\
& \forall \varphi \in \mathcal{C}^{\infty}(\bar{Q}) \text { with }\left.\varphi\right|_{\Sigma}=0 \text { and } \varphi(T)=0 \text { in } \Omega .
\end{aligned}
$$

If we integrate the last integral in this latter identity, we obtain that

$$
\begin{aligned}
\int_{0}^{T} \int_{\Omega}\left(f+u^{\gamma} \chi_{\omega}\right) \varphi d x d t & =-\int_{\Omega} y^{0} \varphi(x, 0) d x+\int_{\Omega} y^{\gamma}(x, 0) \varphi(x, 0) d x \\
& -\left\langle y^{\gamma}, \kappa(\alpha, x) \frac{\partial \varphi}{\partial \nu}\right\rangle_{H^{-3 / 2}\left((0, T) ; H^{-\frac{1}{2}}(\Gamma)\right), H^{3 / 2}\left((0, T) ; H^{\frac{1}{2}}(\Gamma)\right)} \\
& +\int_{0}^{T} \int_{\Omega} \varphi\left(\frac{\partial y^{\gamma}}{\partial t}-\operatorname{div}\left(\kappa(\alpha, x) \nabla y^{\gamma}\right)+a_{0} y^{\gamma}\right) d x d t \\
& \forall \varphi \in \mathcal{C}^{\infty}(\bar{Q}) \text { with }\left.\varphi\right|_{\Sigma}=0 \text { and } \varphi(T)=0 \text { in } \Omega .
\end{aligned}
$$


which in view of (34) yields

$$
\begin{aligned}
0= & \int_{\Omega}\left(-y^{0}+y^{\gamma}(0)\right) \varphi(x, 0) d x \\
- & \left\langle y^{\gamma}, \kappa(\alpha, x) \frac{\partial \varphi}{\partial \nu}\right\rangle_{H^{-3 / 2}\left((0, T) ; H^{-\frac{1}{2}}(\Gamma)\right), H^{3 / 2}\left((0, T) ; H^{\frac{1}{2}}(\Gamma)\right)} \\
& \forall \varphi \in \mathcal{C}^{\infty}(\bar{Q}) \text { with }\left.\varphi\right|_{\Sigma}=0 \text { and } \varphi(T)=0 \text { in } \Omega .
\end{aligned}
$$

If we take in addition $\frac{\partial \varphi}{\partial \nu}=0$ on $\Sigma$, we have $\int_{\Omega}\left(-y^{0}+y^{\gamma}(0)\right) \varphi(x, 0) d x=0$, which implies:

$$
y^{\gamma}(x, 0)=y^{0}(x), \quad x \in \Omega .
$$

It then follows from (35) that

$$
y^{\gamma}=0 \text { on } \Sigma .
$$

Now, combining (34), (36) and (37), we find that $y^{\gamma}=y\left(\alpha, u^{\gamma}, 0\right)$ is solution of (6). This means that

$$
\left\{\begin{aligned}
\frac{\partial y^{\gamma}}{\partial t}-\operatorname{div}\left(\kappa(\alpha, x) \nabla y^{\gamma}\right)+a_{0} y^{\gamma} & =f+u^{\gamma} \chi_{\omega} & & \text { in } Q, \\
y^{\gamma} & =0 & & \text { on } \Sigma, \\
y^{\gamma}(0) & =y^{0} & & \text { in } \Omega .
\end{aligned}\right.
$$

Step 2. We show that $\zeta\left(\alpha, v_{n}\right)$ converges to $\zeta^{\gamma}=\zeta\left(\alpha, u^{\gamma}\right)$ which satisfies (10).

We know that $\zeta_{n}:=\zeta\left(x, t ; \alpha, v_{n}\right)$ is solution of

$$
\left\{\begin{aligned}
-\frac{\partial \zeta_{n}}{\partial t}-\operatorname{div}\left(\kappa(\alpha, x) \nabla \zeta_{n}+a_{0} \zeta_{n}\right. & =-\int_{0}^{1}\left(y_{n}-y(\alpha, 0,0)\right) d \alpha & & \text { in } Q \\
\zeta_{n} & =0 & & \text { on } \Sigma, \\
\zeta_{n}(T) & =0 & & \text { in } \Omega,
\end{aligned}\right.
$$

Therefore, using (26), we can say that there exists a positive constant $C$ independent of $n$ and $\gamma$ such that

$$
\left\|\zeta_{n}\right\|_{L^{2}\left((0, T) ; H^{2}(\Omega)\right)} \leq C
$$

And it follows from the continuity of the trace that

$$
\left\|\frac{\partial \zeta_{n}}{\partial \nu}\right\|_{L^{2}(\Sigma)} \leq C
$$

Consequently,there exists $\zeta^{\gamma} \in L^{2}\left((0, T) ; H^{2}(\Omega)\right)$ and a subsequence extracted from $\left(\zeta\left(\alpha, v_{n}\right)\right)$ (still denoted $\left.\zeta\left(\alpha, v_{n}\right)\right)$ such that

$$
\zeta_{n} \rightarrow \zeta^{\gamma} \text { weakly in } L^{2}\left((0, T) ; H^{2}(\Omega)\right) .
$$


Multiplying the first equation in (39) by $\varphi \in \mathcal{D}(Q)$ and integrating by parts over $Q$,

$$
\int_{Q} \zeta_{n}\left(\frac{\partial \varphi}{\partial t}-\operatorname{div}(\kappa(\alpha, x) \nabla \varphi)+a_{0} \varphi\right) d x d t=-\int_{Q}\left(\int_{0}^{1}\left(y_{n}-y(\alpha, 0,0)\right) d \alpha\right) \varphi d x d t
$$

Passing this latter identity to the limit when $n \rightarrow \infty$ while using (42), (30) and (33), we obtain that

$$
\int_{Q} \zeta^{\gamma}\left(\frac{\partial \varphi}{\partial t}-\operatorname{div}(\kappa(\alpha, x) \nabla \varphi)+a_{0} \varphi\right) d x d t=-\int_{Q}\left(\int_{0}^{1}\left(y^{\gamma}-y(\alpha, 0,0)\right) d \alpha\right) \varphi d x d t
$$

which after integration by parts gives over $Q$,

$\int_{Q}\left(-\frac{\partial \zeta^{\gamma}}{\partial t}-\operatorname{div}\left(\kappa(\alpha, x) \nabla \zeta^{\gamma}\right)+a_{0} \zeta^{\gamma}\right) \varphi d x d t=-\int_{Q}\left(\int_{0}^{1}\left(y^{\gamma}-y(\alpha, 0,0)\right) d \alpha\right) \varphi d x d t$.

Hence, we deduce that

$$
-\frac{\partial \zeta^{\gamma}}{\partial t}-\operatorname{div}\left(\kappa(\alpha, x) \nabla \zeta^{\gamma}\right)+a_{0} \zeta^{\gamma}=-\int_{0}^{1}\left(y^{\gamma}-y(\alpha, 0,0)\right) d \alpha \text { in } Q .
$$

Now, as $\zeta^{\gamma} \in L^{2}\left((0, T), H^{2}(\Omega)\right)$, using (43), we have that $\frac{\partial y^{\gamma}}{\partial t} \in L^{2}\left((0, T), L^{2}(\Omega)\right)$. This implies that $y^{\gamma} \in \mathcal{C}\left([0, T], L^{2}(\Omega)\right)$. On the other hand, we have that $\left.y^{\gamma}\right|_{\Sigma} \in L^{2}(\Sigma)$.

Now, let $\varphi \in \mathcal{C}^{\infty}(\bar{Q})$ with $\left.\varphi\right|_{\Sigma}=0$ and $\varphi(0)=0$ in $\Omega$. If we multiply the first equation in (23) by $\varphi$ and we integrate over $Q$, we obtain:

$$
-\int_{Q}\left(\int_{0}^{1}\left(y_{n}-y(\alpha, 0,0)\right) d \alpha\right) \varphi d x d t=\int_{0}^{T} \int_{\Omega} \zeta_{n}\left(\frac{\partial \varphi}{\partial t}-\operatorname{div}(\kappa(\alpha, x) \nabla \varphi)+a_{0} \varphi\right) d x d t
$$

which by passing to the limit when $n \longrightarrow \infty$ while using (42), (30) and (33) gives

$$
-\int_{Q}\left(\int_{0}^{1}\left(y^{\gamma}-y(\alpha, 0,0)\right) d \alpha\right) \varphi d x d t=\int_{0}^{T} \int_{\Omega} \zeta^{\gamma}\left(\frac{\partial \varphi}{\partial t}-\operatorname{div}(\kappa(\alpha, x) \nabla \varphi)+a_{0} \varphi\right) d x d t,
$$

If we integrate the last integral in this latter identity, we obtain that

$$
\begin{aligned}
-\int_{Q}\left(\int_{0}^{1}\left(y^{\gamma}-y(\alpha, 0,0)\right) d \alpha\right) \varphi d x d t & =\int_{\Omega} \zeta^{\gamma}(T) \varphi(x, T) d x \\
& -\int_{\Sigma} \zeta^{\gamma} \kappa(\alpha, x) \frac{\partial \varphi}{\partial \nu} d \sigma d t \\
& +\int_{0}^{T} \int_{\Omega} \varphi\left(-\frac{\partial \zeta^{\gamma}}{\partial t}-\operatorname{div}\left(\kappa(\alpha, x) \nabla \zeta^{\gamma}\right)+a_{0} \zeta^{\gamma}\right) d x d t \\
& \forall \varphi \in \mathcal{C}^{\infty}(\bar{Q}) \text { with }\left.\varphi\right|_{\Sigma}=0 \text { and } \varphi(0)=0 \text { in } \Omega .
\end{aligned}
$$


which in view of (43) yields

$$
\begin{aligned}
& 0=\int_{\Omega} \zeta^{\gamma}(T) \varphi(x, T) d x-\int_{\Sigma} \zeta^{\gamma} \kappa(\alpha, x) \frac{\partial \varphi}{\partial \nu} d \sigma d t \\
& \forall \varphi \in \mathcal{C}^{\infty}(\bar{Q}) \text { with }\left.\varphi\right|_{\Sigma}=0 \text { and } \varphi(0)=0 \text { in } \Omega \text {. }
\end{aligned}
$$

If we take in addition $\frac{\partial \varphi}{\partial \nu}=0$ on $\Sigma$, we have $\int_{\Omega} \zeta^{\gamma}(T) \varphi(x, T) d x=0$, which implies:

$$
\zeta^{\gamma}(x, T)=0, \quad x \in \Omega .
$$

It the follows from (44) that

$$
\zeta^{\gamma}=0 \text { on } \Sigma .
$$

Now, combining (43), (45) and (46), we find that $\zeta^{\gamma}=\zeta\left(\alpha, u^{\gamma}\right)$ is solution of

$$
\left\{\begin{aligned}
-\frac{\partial \zeta^{\gamma}}{\partial t}-\operatorname{div}\left(\kappa(\alpha, x) \nabla \zeta^{\gamma}\right)+a_{0} \zeta^{\gamma} & =-\int_{0}^{1} \eta\left(\alpha, u^{\gamma}\right) d \alpha & & \text { in } Q, \\
\zeta^{\gamma} & =0 & & \text { on } \Sigma, \\
\zeta^{\gamma}(T) & =0 & & \text { in } \Omega,
\end{aligned}\right.
$$

where as in (11),

$$
\eta\left(\alpha, u^{\gamma}\right)=y\left(\alpha, u^{\gamma}, 0\right)-y(\alpha, 0,0) .
$$

Moreover, using (41), we prove that

$$
\frac{\partial \zeta_{n}}{\partial \nu} \rightarrow \frac{\partial \zeta^{\gamma}}{\partial \nu} \text { weakly in } L^{2}(\Sigma)
$$

In view of $(25)$, there exist $\beta \in L^{2}(\Sigma)$ such that

$$
\frac{\partial \tilde{\zeta}_{n}}{\partial \nu} \rightarrow \beta \text { weakly in } L^{2}(\Sigma)
$$

This means that when $n \rightarrow \infty$,

$$
\int_{\Sigma}\left(\int_{0}^{1} \kappa(\alpha, x) \frac{\partial \zeta_{n}}{\partial \nu} d \alpha\right) \varphi d \sigma d t \rightarrow \int_{\Sigma} \beta \varphi d \sigma d t, \quad \forall \varphi \in L^{2}(\Sigma)
$$

because $\frac{\partial \tilde{\zeta}_{n}}{\partial \nu}=\int_{0}^{1} \kappa(\alpha, x) \frac{\partial \zeta_{n}}{\partial \nu} d \alpha$.

Using (41) and the fact that $\kappa \in C([0,1] \times \bar{\Omega})$, we have that

$$
\left\|\kappa(\alpha, x) \frac{\partial \zeta_{n}}{\partial \nu}\right\|_{L^{2}(\Sigma)} \leq C\left\|\frac{\partial \zeta_{n}}{\partial \nu}\right\|_{L^{2}(\Sigma)} \leq C
$$

Hence it follows from (48) that

$$
\int_{\Sigma}\left(\kappa(\alpha, x) \frac{\partial \zeta_{n}}{\partial \nu}\right) \varphi d \sigma d t \rightarrow \int_{\Sigma}\left(\kappa(\alpha, x) \frac{\partial \zeta^{\gamma}}{\partial \nu}\right) \varphi d \sigma d t, \quad \forall \varphi \in L^{2}(\Sigma) .
$$


Using the Lebesgue dominated convergence theorem and the uniqueness of the limit, we deduce that

$$
\beta=\frac{\partial \tilde{\zeta}^{\gamma}}{\partial \nu}=\int_{0}^{1} \kappa(\alpha, x) \frac{\partial \zeta^{\gamma}}{\partial \nu} d \alpha
$$

Step 3. We prove that $u^{\gamma}$ is unique.

Using the weak lower semi-continuity of the function $J_{\gamma}(v),(30),(32),(28)$, (49) and (53), we deduce that

$$
J_{\gamma}\left(u^{\gamma}\right) \leq \liminf _{n \rightarrow \infty} J_{\gamma}\left(v_{n}\right),
$$

where

$J_{\gamma}\left(u^{\gamma}\right)=\left\|\int_{0}^{1} y\left(\alpha, u^{\gamma}, 0\right) d \alpha-z_{d}\right\|_{L^{2}(Q)}^{2}-J(0,0)+\frac{1}{\gamma}\left\|\frac{\partial \tilde{\zeta}^{\gamma}}{\partial \nu}\right\|_{L^{2}(\Sigma)}^{2}+N\left\|u^{\gamma}\right\|_{L^{2}\left(\omega_{T}\right)}^{2}$.

As $\liminf _{n \rightarrow \infty} J_{\gamma}\left(v_{n}\right)=\lim _{n \rightarrow \infty} J_{\gamma}\left(v_{n}\right)=\inf _{v \in L^{2}\left(\omega_{T}\right)} J_{\gamma}(v)$, we obtain that $u^{\gamma}$ is solution of (20). The strictly convexity of $J_{\gamma}$ allows us to conclude that $u^{\gamma}$ is unique.

We can now characterize the low-regret control $u^{\gamma}$.

Proposition 3.2 Let $u_{\gamma}$ be the solution of (20). Then there exist $p^{\gamma} \in L^{2}(Q)$ and $q^{\gamma} \in Z^{2,1}(Q)$ such that $\left\{y^{\gamma}, \zeta^{\gamma}, p^{\gamma}, q^{\gamma}\right\}$ is solution to

$$
\begin{aligned}
& \left\{\begin{aligned}
\frac{\partial y^{\gamma}}{\partial t}-\operatorname{div}\left(\kappa(\alpha, x) \nabla y^{\gamma}\right)+a_{0} y^{\gamma} & =f+u^{\gamma} \chi_{\omega} & & \text { in } Q \\
y^{\gamma} & =0 & & \text { on } \Sigma \\
y^{\gamma}(0) & =y^{0} & & \text { in } \Omega
\end{aligned}\right.
\end{aligned}
$$

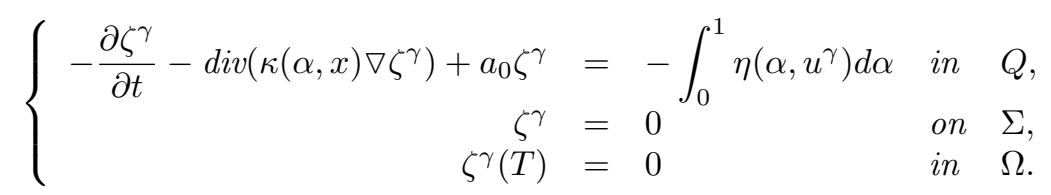

$$
\begin{aligned}
& \left\{\begin{aligned}
\frac{\partial p^{\gamma}}{\partial t}-\operatorname{div}\left(\kappa(\alpha, x) \nabla p^{\gamma}\right)+a_{0} p^{\gamma} & =0 & \text { in } \quad Q, \\
p^{\gamma} & =\frac{1}{\sqrt{\gamma}} \frac{\partial \tilde{\zeta}^{\gamma}}{\partial \nu} & \text { on } \quad \Sigma, \\
p^{\gamma}(0) & =0 & \text { in } \quad \Omega,
\end{aligned}\right. \\
& \left\{\begin{array}{rlrl}
-\frac{\partial q^{\gamma}}{\partial t}-\operatorname{div}\left(\kappa(\alpha, x) \nabla q^{\gamma}\right)+a_{0} q^{\gamma} & =\int_{0}^{1}\left(y^{\gamma}+\frac{p^{\gamma}}{\sqrt{\gamma}}\right) d \alpha-z_{d} & \text { in } \quad Q, \\
q^{\gamma} & =0 & \text { on } \Sigma & \Sigma, \\
q^{\gamma}(T) & =0 & \text { in } \Omega
\end{array}\right.
\end{aligned}
$$

and

$$
u^{\gamma}=-\frac{1}{N} \int_{0}^{1} q^{\gamma} d \alpha \text { in } \omega_{T}
$$

where $q^{\gamma}:=q^{\gamma}\left(\alpha, u^{\gamma}\right)$ and $p^{\gamma}:=p^{\gamma}\left(\alpha, u^{\gamma}\right)$. 
Proof. We have already proved in the Step 1 of Proposition 3.1 that the the state $y^{\gamma}=y\left(\alpha, u^{\gamma}, 0\right)$ associate to optimal control $u^{\gamma}$ satisfies (54). In Step 2, we obtain that $\zeta^{\gamma}=\zeta\left(\alpha, u^{\gamma}\right)$ satisfies (55). To prove (56)-(58), we write the Euler-Lagrange first order optimality condition which characterizes the optimal control $u^{\gamma}$ :

$$
\lim _{\theta \rightarrow 0} \frac{J_{\gamma}\left(u^{\gamma}+\theta v\right)-J_{\gamma}\left(u^{\gamma}\right)}{\theta}=0 \quad \forall v \in L^{2}\left(\omega_{T}\right) .
$$

After some calculations

$$
\begin{aligned}
& \int_{Q}\left(\int_{0}^{1} y\left(\alpha, u^{\gamma}, 0\right) d \alpha-z_{d}\right)\left(\int_{0}^{1} \bar{y}(\alpha, v) d \alpha\right) d x d t \\
& +\frac{1}{\gamma} \int_{\Sigma}\left(\int_{0}^{1} \kappa(\alpha, x) \frac{\partial \bar{\zeta}}{\partial \nu}(\alpha, v) d \alpha\right)\left(\int_{0}^{1} \kappa(\alpha, x) \frac{\partial \zeta}{\partial \nu}\left(\alpha, u^{\gamma}\right) d \alpha\right) d \sigma d t \\
& +N \int_{\omega_{T}} u^{\gamma} v d x d t=0 \quad \forall v \in L^{2}\left(\omega_{T}\right),
\end{aligned}
$$

where $\bar{y}(\alpha, v)$ is solution to

$$
\left\{\begin{array}{rlll}
\frac{\partial \bar{y}}{\partial t}-\operatorname{div}(\kappa(\alpha, x) \nabla \bar{y})+a_{0} \bar{y} & =v \chi_{\omega} & \text { in } Q, \\
\bar{y} & =0 & & \text { on } \Sigma, \\
\bar{y}(0) & =0 & & \text { in } \Omega,
\end{array}\right.
$$

and $\bar{\zeta}(\alpha, v)$ is solution to

$$
\left\{\begin{array}{rlrl}
-\frac{\partial \bar{\zeta}}{\partial t}-\operatorname{div}(\kappa(\alpha, x) \nabla \bar{\zeta})+a_{0} \bar{\zeta} & =-\int_{0}^{1} \bar{y}(\alpha, v) d \alpha & \text { in } Q \\
\bar{\zeta} & =0 & & \text { on } \Sigma, \\
\bar{\zeta}(T) & =0 & & \text { in } \Omega .
\end{array}\right.
$$

Now, if we multiply the first equation in (61) and in (62) by $\int_{0}^{1} q^{\gamma}\left(\alpha, u^{\gamma}\right) d \alpha \in L^{2}(Q)$ and $\frac{1}{\sqrt{\gamma}} \int_{0}^{1} p^{\gamma}\left(\alpha, u^{\gamma}\right) d \alpha$ respectively, then integrate by parts over $Q$, we obtain

$$
\begin{aligned}
& \int_{Q}\left[\left(\int_{0}^{1} \bar{y}(\alpha, v) d \alpha\right)\left(\int_{0}^{1}\left(y\left(\alpha, u^{\gamma}, 0\right)+\frac{1}{\sqrt{\gamma}} p^{\gamma}\left(\alpha, u^{\gamma}\right)\right) d \alpha-z_{d}\right)\right] d x d t \\
= & \int_{\omega_{T}} v\left(\int_{0}^{1} q^{\gamma}\left(\alpha, u^{\gamma}\right) d \alpha\right) d x d t,
\end{aligned}
$$

and

$$
\begin{aligned}
& \int_{\Sigma}\left[-\frac{1}{\gamma} \int_{0}^{1}\left(\kappa(\alpha, x) \frac{\partial \bar{\zeta}}{\partial \nu}(\alpha, v)\right) d \alpha\right]\left[\int_{0}^{1} \kappa(\alpha, x) \frac{\partial \zeta}{\partial \nu}\left(\alpha, u^{\gamma}\right) d \alpha\right] d \sigma d t \\
= & \int_{Q}\left[\int_{0}^{1} \bar{y}(\alpha, v) d \alpha\right]\left[\frac{1}{\sqrt{\gamma}} \int_{0}^{1} p^{\gamma} d \alpha\right] d x d t .
\end{aligned}
$$


Combining (60), (63) and (64), we have that

$$
\int_{\omega_{T}}\left(N u^{\gamma}+\int_{0}^{1} q^{\gamma}\left(\alpha, u^{\gamma}\right) d \alpha\right) v d x d t=0, \quad \forall v \in L^{2}\left(\omega_{T}\right),
$$

which implies that

$$
N u^{\gamma}+\int_{0}^{1} q^{\gamma}\left(\alpha, u^{\gamma}\right)=0 \text { in } \omega_{T}
$$

\section{Existence and characterization of the no-regret control}

Proposition 4.1 The low-regret control $u^{\gamma}$ converges in $L^{2}(Q)$ to the no-regret control $\hat{u}$, solution of (18).

Proof. From (20), we have that

$$
J_{\gamma}\left(u^{\gamma}\right) \leq J_{\gamma}(0)=0,
$$

which in view of the expression of $J_{\gamma}$ given by (21) imples that

$$
\left\|\int_{0}^{1} y^{\gamma} d \alpha-z_{d}\right\|_{L^{2}(Q)}^{2}+N\left\|u^{\gamma}\right\|_{L^{2}\left(\omega_{T}\right)}^{2}+\frac{1}{\gamma}\left\|\frac{\partial \tilde{\zeta}^{\gamma}}{\partial \nu}\right\|_{L^{2}(\Sigma)}^{2} \leq J(0,0) .
$$

Hence, we deduce that

$$
\begin{aligned}
\left\|u^{\gamma}\right\|_{L^{2}\left(\omega_{T}\right)} & \leq \frac{\sqrt{J(0,0)}}{\sqrt{N}}, \\
\left\|\frac{\partial \tilde{\zeta}^{\gamma}}{\partial \nu}\right\|_{L^{2}(\Sigma)} & \leq \sqrt{J(0,0)} \sqrt{\gamma}, \\
\left\|\int_{0}^{1} y^{\gamma}(\alpha, ., .) d \alpha\right\|_{L^{2}(Q)} & \leq \sqrt{J(0,0)}+\left\|z_{d}\right\|_{L^{2}(Q)} .
\end{aligned}
$$

In view of (69) and (54), there exists $C>0$ independent of $\gamma$ such that

$$
\left\|y^{\gamma}\right\|_{L^{2}\left((0, T) ; H_{0}^{1}(\Omega)\right)} \leq C .
$$

Thus, there exist $\hat{u} \in L^{2}\left(\omega_{T}\right), \hat{y} \in L^{2}\left((0, T) ; H_{0}^{1}(\Omega)\right)$ such that

$$
\begin{array}{lll}
u^{\gamma} \rightarrow \hat{u} & \text { weakly in } L^{2}\left(\omega_{T}\right), \\
y^{\gamma} \rightarrow \hat{y} & \text { weakly in } L^{2}\left((0, T) ; H_{0}^{1}(\Omega)\right) .
\end{array}
$$


Using (73), (74) and (54), we show as in pages 9-11 that $\hat{y}=y(\alpha, \hat{u}, 0)$ satisfies

$$
\begin{aligned}
\frac{\partial \hat{y}}{\partial t}-\operatorname{div}(\kappa(\alpha, x) \nabla \hat{y})+a_{0} \hat{y} & =f+\hat{u} \chi_{\omega} & & \text { in } Q, \\
\hat{y} & =0 & & \text { on } \Sigma, \\
\hat{y}(0) & =y^{0} & & \text { in } \Omega .
\end{aligned}
$$

Moreover, using (71), (74) and the Lebesgue dominated convergence theorem, we have that

$$
\int_{0}^{1} y\left(\alpha, u^{\gamma}, 0\right) d \alpha \rightarrow \int_{0}^{1} y(\alpha, \hat{u}, 0) d \alpha \text { weakly in } L^{2}(Q) .
$$

From (71) and (55), we prove that there exists a positive constant $C$ such that

$$
\left\|\zeta\left(\alpha, u^{\gamma}\right)\right\|_{L^{2}\left((0, T) ; H^{2}(\Omega)\right)} \leq C .
$$

Therefore the continuity of the trace allows us to write that

$$
\left\|\frac{\partial \zeta^{\gamma}}{\partial \nu}\right\|_{L^{2}(\Sigma)} \leq C
$$

Consequently, there exists $\hat{\zeta} \in L^{2}\left((0, T) ; H^{2}(\Omega)\right)$ such that

$$
\zeta\left(\alpha, u^{\gamma}\right) \rightarrow \hat{\zeta} \text { weakly in } L^{2}\left((0, T) ; H^{2}(\Omega)\right) .
$$

Therefore proceeding as for $\zeta_{n}$ in pages 11-14, we prove that $\hat{\zeta}=\zeta(\alpha, \hat{u})$ is solution to

$$
\left\{\begin{aligned}
-\frac{\partial \hat{\zeta}}{\partial t}-\operatorname{div}(\kappa(\alpha, x) \nabla \hat{\zeta})+a_{0} \hat{\zeta} & =-\int_{0}^{1}(\hat{y}-y(\alpha, 0,0)) d \alpha & & \text { in } \quad Q, \\
\hat{\zeta} & =0 & & \text { on } \Sigma \\
\hat{\zeta}(T) & =0 & & \text { in } \quad \Omega .
\end{aligned}\right.
$$

Moreover, using (78), we have the following

$$
\frac{\partial \zeta^{\gamma}}{\partial \nu} \rightarrow \frac{\partial \hat{\zeta}}{\partial \nu} \quad \text { weakly in } \quad L^{2}(\Sigma)
$$

In view of (70),

$$
\frac{\partial \tilde{\zeta}^{\gamma}}{\partial \nu} \rightarrow 0 \text { strongly in } L^{2}(\Sigma)
$$

Because $\frac{\partial \tilde{\zeta}^{\gamma}}{\partial \nu}=\int_{0}^{1} \kappa(\alpha, x) \frac{\partial \zeta^{\gamma}}{\partial \nu} d \alpha$. Using (78) and the fact that $\kappa \in C([0,1] \times \bar{\Omega})$, we have that

$$
\left\|\kappa(\alpha, x) \frac{\partial \zeta^{\gamma}}{\partial \nu}\right\|_{L^{2}(\Sigma)} \leq C\left\|\frac{\partial \zeta_{n}}{\partial \nu}\right\|_{L^{2}(\Sigma)} \leq C .
$$


Hence it follows from (81) that

$$
\int_{\Sigma}\left(\kappa(\alpha, x) \frac{\partial \zeta^{\gamma}}{\partial \nu}\right) \varphi d \sigma d t \longrightarrow \int_{\Sigma}\left(\kappa(\alpha, x) \frac{\partial \hat{\zeta}}{\partial \nu}\right) \varphi d \sigma d t, \quad \forall \varphi \in L^{2}(\Sigma) .
$$

Using Lebesgue dominated convergence theorem and (82), we deduce that

$$
0=\int_{0}^{1} \kappa(\alpha, x) \frac{\partial \hat{\zeta}}{\partial \nu} d \alpha
$$

which in view of (19) allows to say that $\hat{u} \in \mathcal{O}$. Therefore, we conclude that the low-regret control $u^{\gamma}$ converges to the no-regret control $\hat{u}$.

Proposition 4.2 The no-regret control $\hat{u}$, solution of (5) (or equivalently (18)), is characterized by following optimality system:

$$
\begin{aligned}
& \left\{\begin{aligned}
\frac{\partial \hat{y}}{\partial t}-\operatorname{div}(\kappa(\alpha, x) \nabla \hat{y})+a_{0} \hat{y} & =f+\hat{u} \chi_{\omega} & \text { in } & Q \\
\hat{y} & =0 & & \text { on } \Sigma \\
\hat{y}(0) & =y^{0} & & \text { in } \Omega
\end{aligned}\right. \\
& \left\{\begin{aligned}
-\frac{\partial \hat{\zeta}}{\partial t}-\operatorname{div}(\kappa(\alpha, x) \nabla \hat{\zeta})+a_{0} \hat{\zeta} & =-\int_{0}^{1}(\hat{y}-y(\alpha, 0,0)) d \alpha & & \text { in } \quad Q \\
\hat{\zeta} & =0 & & \text { on } \Sigma \\
\hat{\zeta}(T) & =0 & & \text { in } \Omega
\end{aligned}\right. \\
& \left\{\begin{array}{rllll}
\frac{\partial \hat{p}}{\partial t}-\operatorname{div}(\kappa(\alpha, x) \nabla \hat{p})+a_{0} \hat{p} & =0 & \text { in } & Q \\
\hat{p} & = & \lambda_{1} & \text { on } & \Sigma \\
\hat{p}(0) & = & 0 & \text { in } & \Omega
\end{array}\right. \\
& \left\{\begin{aligned}
-\frac{\partial \hat{q}}{\partial t}-\operatorname{div}(\kappa(\alpha, x) \nabla \hat{q})+a_{0} \hat{q} & =\int_{0}^{1}\left(\hat{y}+\lambda_{2}\right) d \alpha-z_{d} & & \text { in } Q \\
\hat{q} & =0 & & \text { on } \Sigma, \\
\hat{q}(T) & =0 & & \text { in } \Omega
\end{aligned}\right.
\end{aligned}
$$

and

$$
\hat{u}=-\frac{1}{N} \int_{0}^{1} \hat{q} d \alpha \quad \text { in } \omega_{T}
$$

where $\lambda_{1}=\lim _{\gamma \rightarrow 0} \frac{1}{\sqrt{\gamma}} \frac{\partial \tilde{\zeta}^{\gamma}}{\partial \nu}$ and $\lambda_{2}=\lim _{\gamma \rightarrow 0} \frac{1}{\sqrt{\gamma}} \int_{0}^{1} p^{\gamma}\left(\alpha, u^{\gamma}\right) d \alpha$.

Proof. we have already relations (86) and (87) ( see (75) and (80) ). The proof of (88)- (90) is obtained by passing to the limit in (56) - (58). To this end we will proceed in 3 steps.

Step 1. We show that $\hat{p}$ satisfies (88). 
In view of (70), we have that there exists a positive constant $C$ such that:

$$
\left\|\frac{1}{\sqrt{\gamma}} \frac{\partial \tilde{\zeta}^{\gamma}}{\partial \nu}\right\|_{L^{2}(\Sigma)} \leq C
$$

which in view of (56) allows us to say that

$$
\left\|p^{\gamma}\right\|_{L^{2}(Q)} \leq C
$$

Hence, there exists $\lambda_{1} \in L^{2}(\Sigma)$ and $\hat{p} \in L^{2}(Q)$ such that:

$$
\begin{aligned}
\frac{1}{\sqrt{\gamma}} \frac{\partial \tilde{\zeta}^{\gamma}}{\partial \nu} & \rightarrow \lambda_{1} \quad \text { weakly in } L^{2}(\Sigma) \\
p^{\gamma} & \rightarrow \hat{p} \quad \text { weakly in } L^{2}(Q) .
\end{aligned}
$$

If we multiply the first equation in (56) by $\varphi \in \mathcal{D}(Q)$, we get

$$
\begin{aligned}
0 & =\int_{Q}\left(\frac{\partial p^{\gamma}}{\partial t}-\operatorname{div}\left(\kappa(\alpha, x) \nabla p^{\gamma}\right)+a_{0} p^{\gamma}\right) \varphi d x d t \\
& =\int_{Q} p^{\gamma}\left(-\frac{\partial \varphi}{\partial t}-\operatorname{div}(\kappa(\alpha, x) \nabla \varphi)+a_{0} \varphi\right) d x d t .
\end{aligned}
$$

Passing this latter identity to the limit when $\gamma \rightarrow 0$ while using (94), we deduce that

$$
0=\int_{Q} \hat{p}\left(-\frac{\partial \varphi}{\partial t}-\operatorname{div}(\kappa(\alpha, x) \nabla \varphi)+a_{0} \varphi\right) d x d t
$$

which after integration by parts gives

$$
0=\int_{Q}\left(\frac{\partial \hat{p}}{\partial t}-\operatorname{div}(\kappa(\alpha, x) \nabla \hat{p})+a_{0} \hat{p}\right) \varphi d x d t
$$

from which we get,

$$
\frac{\partial \hat{p}}{\partial t}-\operatorname{div}(\kappa(\alpha, x) \nabla \hat{p})+a_{0} \hat{p}=0 \text { in } Q .
$$

As $\hat{p} \in L^{2}(Q)$ and $\frac{\partial \hat{p}}{\partial t} \in L^{2}\left((0, T), H^{-2}(\Omega)\right)$ we have that $\hat{p} \in \mathcal{C}\left([0, T], H^{-1}(\Omega)\right)$.On the other hand, $\hat{p} \in L^{2}(Q)$ and $\operatorname{div}(\kappa(\alpha, x) \nabla \hat{p}) \in H^{-1}\left((0, T), L^{2}(\Omega)\right)$. This implies that that $\left.\hat{p}\right|_{\Sigma}$ and $\left.\frac{\partial \hat{p}}{\partial \nu}\right|_{\Sigma}$ exist and belong respectively to $H^{-1}\left((0, T) ; H^{-\frac{1}{2}}(\Gamma)\right)$ and $H^{-1}\left((0, T) ; H^{-\frac{3}{2}}(\Gamma)\right)$.

Now, let $\varphi \in \mathcal{C}^{\infty}(\bar{Q})$ with $\left.\varphi\right|_{\Sigma}=0$ and $\varphi(T)=0$ in $\Omega$. If we multiply the first equation in (56) by $\varphi$ and we integrate over $Q$, we obtain

$$
\int_{\Sigma} \frac{1}{\sqrt{\gamma}} \frac{\partial \tilde{\zeta}^{\gamma}}{\partial \nu} \kappa(\alpha, x) \frac{\partial \varphi}{\partial \nu} d \sigma d t+\int_{Q} p^{\gamma}\left(-\frac{\partial \varphi}{\partial t}-\operatorname{div}\left(\kappa(\alpha, x) \nabla \varphi+a_{0} \varphi\right)\right) d x d t=0
$$


Passing this latter identity to the limit when $\gamma \longrightarrow 0$ while using (93) and (94), we obtain

$$
\begin{aligned}
& \int_{\Sigma} \lambda_{1} \kappa(\alpha, x) \frac{\partial \varphi}{\partial \nu}+\int_{Q} \hat{p}\left(-\frac{\partial \varphi}{\partial t}-\operatorname{div}\left(\kappa(\alpha, x) \nabla \varphi+a_{0} \varphi\right)\right) d x d t=0, \\
& \forall \varphi \in \mathcal{C}^{\infty}(\bar{Q}) \text { such that }\left.\varphi\right|_{\Sigma}=0 \text { and } \varphi(T)=0,
\end{aligned}
$$

which after integration by parts yields

$$
\begin{array}{r}
\int_{\Sigma} \kappa(\alpha, x) \lambda_{1} \frac{\partial \varphi}{\partial \nu}+\langle\hat{p}(0), \varphi(0)\rangle_{H^{-1}(\Omega), H_{0}^{1}(\Omega)}- \\
\left\langle\hat{p}, \kappa(\alpha, x) \frac{\partial \varphi}{\partial \nu}\right\rangle_{H^{-3 / 2}\left((0, T) ; H^{-\frac{1}{2}}(\Gamma)\right), H^{3 / 2}\left((0, T) ; H^{\frac{1}{2}}(\Gamma)\right)}+ \\
\int_{Q}\left(\frac{\partial \hat{p}}{\partial t}-\operatorname{div}\left(\kappa(\alpha, x) \nabla \hat{p}+a_{0} \hat{p}\right)\right) \varphi d x d t=0 \\
\forall \varphi \in \mathcal{C}^{\infty}(\bar{Q}) \text { such that }\left.\varphi\right|_{\Sigma}=0 \text { and } \varphi(T)=0 .
\end{array}
$$

In view of $(95)$, we deduce that

$$
\begin{array}{r}
\langle\hat{p}(0), \varphi(0)\rangle_{H^{-1}(\Omega), H_{0}^{1}(\Omega)}- \\
\left\langle\left(\lambda_{1}-\hat{p}\right), \kappa(\alpha, x) \frac{\partial \varphi}{\partial \nu}\right\rangle_{H^{-3 / 2}\left((0, T) ; H^{-\frac{1}{2}}(\Gamma)\right), H^{3 / 2}\left((0, T) ; H^{\frac{1}{2}}(\Gamma)\right)}=0, \\
\forall \varphi \in \mathcal{C}^{\infty}(\bar{Q}) \text { such that }\left.\varphi\right|_{\Sigma}=0 \text { and } \varphi(T)=0 .
\end{array}
$$

If we take in addition $\frac{\partial \varphi}{\partial \nu}=0$ on $\Sigma$, we have

$$
\int_{\Omega} \hat{p}(0) \varphi(0) d x=0
$$

which implies that

$$
\hat{p}(0)=0 \text { in } \Omega,
$$

and it follows from (96) that

$$
\int_{\Sigma}\left(\lambda_{1}-\hat{p}\right) \kappa(\alpha, x) \frac{\partial \varphi}{\partial \nu}=0 .
$$

This implies

$$
\hat{p}=\lambda_{1} \quad \text { on } \quad \Sigma .
$$

Combining (95), (97) and (98), we have that $\hat{p}$ satisfies (88) .

Step 2. We show that $q^{\gamma}$ converge towards $\hat{q}$ which satisfies (89). Let $q_{1}^{\gamma}$ and $q_{2}^{\gamma}$ be respectively solution of

$$
\left\{\begin{aligned}
-\frac{\partial q_{1}^{\gamma}}{\partial t}-\operatorname{div}\left(\kappa(\alpha, x) \nabla q_{1}^{\gamma}\right)+a_{0} q_{1}^{\gamma} & =\int_{0}^{1} y^{\gamma} d \alpha-z_{d} & & \text { in } Q \\
q_{1}^{\gamma} & =0 & & \text { on } \Sigma, \\
q_{1}^{\gamma}(T) & =0 & & \text { in } \Omega,
\end{aligned}\right.
$$


and

$$
\left\{\begin{aligned}
-\frac{\partial q_{2}^{\gamma}}{\partial t}-\operatorname{div}\left(\kappa(\alpha, x) \nabla q_{2}^{\gamma}\right)+a_{0} q_{2}^{\gamma} & =\int_{0}^{1} \frac{p^{\gamma}}{\sqrt{\gamma}} d \alpha & & \text { in } Q, \\
q_{2}^{\gamma} & =0 & & \text { on } \Sigma, \\
q_{2}^{\gamma}(T) & =0 & & \text { in } \Omega .
\end{aligned}\right.
$$

Then $q_{1}^{\gamma}+q_{2}^{\gamma}=q^{\gamma}$, solution of (57). According to (71), there exists a constant $C>0$ independent of $\gamma$ such

$$
\left\|q_{1}^{\gamma}\right\|_{L^{2}\left((0, T) ; H^{2}(\Omega)\right)} \leq C .
$$

Therefore there exists $\hat{q}_{1} \in L^{2}\left((0, T) ; H^{2}(\Omega)\right)$ such that

$$
q_{1}^{\gamma} \rightarrow \hat{q}_{1} \text { weakly in } L^{2}\left((0, T) ; H^{2}(\Omega)\right) .
$$

Proceeding as for $\zeta_{n}$ in pages 11-14, we prove using (102) and (76) that $\hat{q}_{1}$ is solution to

$$
\left\{\begin{array}{rlrl}
-\frac{\partial \hat{q}_{1}}{\partial t}-\operatorname{div}\left(\kappa(\alpha, x) \nabla \hat{q}_{1}+a_{0} \hat{q}_{1}\right. & =\int_{0}^{1} \hat{y} d \alpha-z_{d} & & \text { in } Q, \\
\hat{q}_{1} & =0 & & \text { on } \Sigma, \\
\hat{q}_{1}(T) & =0 & \text { in } \Omega .
\end{array}\right.
$$

To complete the proof of the convergence of $q_{\theta}^{\gamma}$, we need to prove that prove that $\left\|\int_{0}^{1} \frac{p^{\gamma}}{\sqrt{\gamma}} d \alpha\right\|_{L^{2}(Q)} \leq C$ for some $C>0$ independent of $\gamma$.

We consider the following set

$$
\mathcal{E}=\left\{\Phi(v)=\int_{0}^{1} \bar{y}(\alpha, v, 0) d \alpha \mid v \in L^{2}\left(\omega_{T}\right)\right\} .
$$

In view of Remark $1, \mathcal{E} \subset L^{2}(Q)$. We define on $\mathcal{E} \times \mathcal{E}$ the inner product:

$$
\begin{aligned}
& \langle\Phi(v), \phi(w)\rangle_{\mathcal{E}}=\int_{Q} v w d x d t+\int_{Q}\left(\int_{0}^{1} \bar{y}(\alpha, v, 0) d \alpha\right)\left(\int_{0}^{1} \bar{y}(\alpha, w, 0) d \alpha\right) d x d t \\
& \forall \Phi(v), \phi(w) \in \mathcal{E} .
\end{aligned}
$$

Then $\mathcal{E}$ endowed with the norm

$$
\|\Phi(v)\|_{\mathcal{E}}^{2}=\int_{Q}|v|^{2} d x d t+\int_{Q}\left(\int_{0}^{1} \bar{y}(\alpha, v, 0) d \alpha\right)^{2} d x d t, \forall \Phi(v) \in \mathcal{E}
$$

Is an Hilbert space. Now if we combine (60) and (64), we obtain that

$$
\begin{aligned}
& \int_{Q}\left[\int_{0}^{1} \bar{y}(\alpha, v) d \alpha\right]\left[\frac{1}{\sqrt{\gamma}} \int_{0}^{1} p^{\gamma} d \alpha\right] d x d t=N \int_{\omega_{T}} u^{\gamma} v d x d t+ \\
& \int_{Q}\left(\int_{0}^{1} y\left(\alpha, u^{\gamma}, 0\right) d \alpha-z_{d}\right)\left(\int_{0}^{1} \bar{y}(\alpha, v) d \alpha\right) d x d t \quad \forall v \in L^{2}\left(\omega_{T}\right) .
\end{aligned}
$$


We set $T_{\gamma}\left(u^{\gamma}\right)=\frac{1}{\sqrt{\gamma}} \int_{0}^{1} p^{\gamma} d \alpha$. Then in view of (107),

$$
\begin{aligned}
\int_{Q} T_{\gamma}\left(u^{\gamma}\right) \Phi(v) d x d t & =N \int_{Q} u^{\gamma} v d x d t \\
& +\int_{\substack{Q \\
\forall}}\left(\int_{0}^{1} y\left(\alpha, u^{\gamma}, 0\right) d \alpha-z^{2}(Q) .\right.
\end{aligned}
$$

Observing that there exists a constant $C$ independent of $\gamma$ such that

$$
\begin{aligned}
& \left|\int_{Q}\left(\int_{0}^{1} y\left(\alpha, u^{\gamma}, 0\right) d \alpha-z_{d}\right)\left(\int_{0}^{1} \bar{y}(\alpha, v) d \alpha\right) d x d t+N \int_{Q} u^{\gamma} v d x d t\right| \leq \\
& \left(\left\|\int_{0}^{1} y\left(\alpha, u^{\gamma}, 0\right) d \alpha-z_{d}\right\|_{L^{2}(Q)}^{2}+N^{2}\left\|u^{\gamma}\right\|_{L^{2}(Q)}^{2}\right)^{1 / 2}\|\Phi(v)\|_{\mathcal{E}} \leq C\|\Phi(v)\|_{\mathcal{E}}
\end{aligned}
$$

because of (69) and (71). We then deduce from (108) that

$$
\left|\int_{Q} T_{\gamma}\left(u^{\gamma}\right) \Phi(v) d x d t\right| \leq C\|\Phi(v)\|_{\mathcal{E}}
$$

This means that

$$
\left\|T_{\gamma}\left(u^{\gamma}\right)\right\|_{\mathcal{E}}=\left\|\frac{1}{\sqrt{\gamma}} \int_{0}^{1} p^{\gamma} d \alpha\right\|_{\mathcal{E}} \leq C
$$

In particular

$$
\left\|\frac{1}{\sqrt{\gamma}} \int_{0}^{1} p^{\gamma} d \alpha\right\|_{L^{2}(Q)} \leq C .
$$

This implies that

$$
\left\|q_{2}^{\gamma}\right\|_{L^{2}\left((0, T) ; H^{2}(\Omega)\right)} \leq C .
$$

Therefore there exist $\hat{q}_{2} \in L^{2}\left((0, T) ; H^{2}(\Omega)\right) \lambda_{2} \in L^{2}(Q)$ such that

$$
\begin{aligned}
q_{2}^{\gamma} & \rightarrow \hat{q}_{2} \text { weakly in } L^{2}\left((0, T) ; H^{2}(\Omega)\right), \\
\frac{1}{\sqrt{\gamma}} \int_{0}^{1} p^{\gamma} d \alpha & \rightarrow \lambda_{2} \text { weakly in } L^{2}(Q) .
\end{aligned}
$$

Proceeding as for $\zeta_{n}$ in pages 11-14, we prove using (111) and (112) that $\hat{q}_{2}$ is solution to

$$
\left\{\begin{array}{lllll}
-\frac{\partial \hat{q}_{2}}{\partial t}-\operatorname{div}\left(\kappa(\alpha, x) \nabla \hat{q}_{2}\right)+a_{0} \hat{q}_{2} & = & \lambda_{2} & \text { in } & Q, \\
\hat{q}_{2} & = & 0 & \text { on } & \Sigma, \\
\hat{q}_{2}(T) & = & 0 & \text { in } & \Omega .
\end{array}\right.
$$

From (101) and (110), we have that $q^{\gamma}=q_{1}^{\gamma}+q_{2}^{\gamma}$ is such that

$$
\left\|q^{\gamma}\right\|_{L^{2}(Q)} \leq C
$$


for some $C>0$ independent of $\gamma$. Hence,

$$
q^{\gamma} \rightarrow \hat{q}_{1}+\hat{q}_{2}=\hat{q} \text { weakly in } L^{2}(Q)
$$

and $\hat{q}$ satisfies (89).

Step 3. Using Remark 2 and (114), we have that

$$
\left\|\int_{0}^{1} q^{\gamma} d \alpha\right\|_{L^{2}(Q)} \leq C .
$$

and using Lebesgue dominated convergence theorem we prove that

$$
\int_{0}^{1} q^{\gamma} d \alpha \rightarrow \int_{0}^{1} q d \alpha \text { weakly in } L^{2}(Q) .
$$

Finally passing to the limits in (66) while using (73) and (117), we deduce (90).

\section{Concluding remarks}

In this paper, we proved that averaging the cost function related to our model, the system is still controllable and gives an optimal control which does not depends of the unknown parameter. Using the Lebesgue dominated convergence theorem, we remark also that as $\gamma \rightarrow 0$, the limits of the average state is not far from the average of the limit of the corresponding original state.

Acknowledgment: The first author was supported by the Alexander von Humboldt foundation, under the programme financed by the BMBF entitled "German research Chairs". The second and the third authors are grateful for the facilities provided by the German research Chairs.

\section{References}

[1] J.L. Lions, Contrôle de Pareto de systèmes distribués. Le cas stationnaire, C. R. Acad. Sci. Paris Sér. I 302 (6)(1986) 223-227.

[2] J.L. Lions, Contrôle à moindres regrets des systèmes distribués, C. R. Acad. Sci. Paris Sér. I 315 (1992)1253-1257.

[3] J.L. Lions, No-regret and low-regret control. Environment, economics and their mathematical models, 1994.

[4] O. Nakoulima, A. Omrane, J. Velin, Perturbations à moindres regrets dans les systèmes distribués à données manquantes, C. R. Acad. Sci. Paris Sr. I 330 (2000) 801806.

[5] Dorville R, O. Nakoulima, Omrane A., Low-regret control for singular distributedsystems: the backwards heat ill posed problem, Applied Math. Letters, 1715, pp 549-552 (2004) 
[6] Dorville R, Nakoulima O., Omrane A., Contrôle optimal pour les problèmes de contrôlabilité des systèmesdistribués à données manquantes, C.R. Acad. Sci. Paris, Ser. I Math., Vol 338, pp 921 - 924 (2004)

[7] J. L. Lions, Quelques notions dans l'analyse et le contrôle des systèmes à données incomplètes, Procedings of the XI th congress on Differential Equations and Applications

[8] Lions J.L., Magenes E.: Problèmes aux limites non homogènes et applications. Dunod, Paris (1968)

[9] J.L Lions. Contrôle à moindres regrets des systèmes distribués. C.R.Acad.Sci. Paris,Ser.I Math.315,1253-1257(1992).

[10] O. Nakoulima, A. Omrane, R. Dorville. Low-regret control of singular distributed systems: the ill-posed backwards heat problem. Appl. Math. Lett. 17, 549-552 (2004).

[11] O. Nakoulima,A. Omrane, J. Velin. No-regret control for nonlinear distributed systems with incomplete data. J. Math. Pures Appl. 81, 1161-1189 (2002).

[12] B. Jacob, A. Omrane. Optimal control for age-structured population dynamics of incomplete data. J. Math. Anal. Appl.370(1), 42-48 (2010).

[13] O. Nakoulima, A. Omrane, J. Velin. Low-regret perturbations in distributed systems with incomplete data. SIAM J. Control Optim. 42(4), 1167-1184 (2003).

[14] G. Mophou. Optimal control for fractional diffusion equations with incomplete data. J. Optim. Theory Appl. (2015).

[15] D. Baleanu, C. Joseph and G. Mophou G. Low-regret control for a fractional wave equation with incomplete data. Adv Differ Equ (2016) 2016: 240. https://doi.org/10.1186/s13662-016-0970-8

[16] E. Zuazua.Averaged control. Automatica 50(2014)3077-3087.

[17] J. Lohéac and E. Zuazua Averaged controllability of parameter dependent conservative semigroups. J. DifferentialEquations. 262(2017)15401574.

[18] M. Lazar and E. Zuazua.Averaged control and observation of parameterdepending wave equations, C. R. Math. Acad. Sci. Paris 352(6) (2014) 497502 .

[19] Q. Lü and E. Zuazua. Averaged controllability for random evolution partial differential equations. J.Math. Pures Appl. (9) 105(3) (2016) 367414. 